\title{
Contaminantes atmosféricos y su correlación con infecciones agudas de las vías respiratorias en niños de Guadalajara, Jalisco
}

\author{
Hermes Ulises Ramírez-Sánchez, Dr en C,(1) María Dolores Andrade-García, MCSP,(2) \\ Miguel Ernesto González-Castañeda, Dr en C, ${ }^{(3)}$ Alfredo de Jesús Celis-de la Rosa, Dr en E. ${ }^{(3)}$
}

\begin{abstract}
Ramírez-Sánchez HU,Andrade-García MD, González-Castañeda ME, Celis-De la Rosa AJ. Contaminantes atmosféricos y su correlación con infecciones agudas de las vías respiratorias en niños de Guadalajara, Jalisco Salud Publica Mex 2006;48:385-394.
\end{abstract}

\begin{abstract}
Resumen
Objetivo. Describir la relación entre los niveles de concentración de los contaminantes atmosféricos y el número de consultas por infecciones agudas de las vías respiratorias en niños menores de cinco años del IMSS, ISSSTE y SS] del área urbana de Guadalajara, en el lapso de 2000 a 2002. Material y métodos. Se realizó un estudio ecológico transversal para describir la correlación entre las modas promedio mensuales, medias móviles mensuales interpoladas de los contaminantes (monóxido de carbono, dióxido de nitrógeno, ozono, partículas menores de 10 micras y dióxido de azufre) y las consultas mensuales registradas por infecciones agudas de las vías respiratorias en niños menores de cinco años. Resultados. Los contaminantes atmosféricos monóxido de carbono y dióxido de nitrógeno muestran correlación significativa con las infecciones agudas de las vías respiratorias en niños menores de cinco años del área urbana de Guadalajara. Las correlaciones presentadas fueron: $\mathrm{CO}(r=0.05)$ y NO$(r=0.09)$. Conclusiones. Pese a que las concentraciones de contaminantes se mantienen por debajo de la norma oficial, los contaminantes monóxido de carbono y dióxido de nitrógeno inciden en la salud de la población infantil del área urbana de Guadalajara. Aunque las modas mensuales y medias móviles mensuales no rebasan la normatividad establecida, sí lo hacen los máximos registrados; esto permite indicar que si bien en la ma-
\end{abstract}

Ramírez-Sánchez HU, Andrade-García MD, González-Castañeda ME, Celis-De la Rosa AJ.

Air pollutants and their correlation with medical visits for acute respiratory infections in children less than five years of age in urban Guadalajara, Mexico. Salud Publica Mex 2006;48:385-394.

\begin{abstract}
Objective: To describe the correlation between the concentration levels of atmospheric air pollutants and the number of medical visits to IMSS, ISSSTE and of SSJ healthcare facilities of the Urban Area of Guadalajara between 2000 2002 by children under five years suffering from acute respiratory infections. Material and Methods: An ecological study was performed to describe the correlation between the interpolated monthly average modes, monthly mobile average of air pollutants: carbon monoxide, nitrogen dioxide, ozone, and particles with an aerodynamic diameter of less than 10 microns $\left(\mathrm{PM}_{10}\right)$ and sulfur dioxide, and the number of medical visits per month due to acute respiratory infections in children under five years. Results: The air pollutants: carbon monoxide and nitrogen dioxide show a significant correlation with the incidence of acute respiratory infections in children less than five years of age in the Urban Area of Guadalajara. The correlation coefficients were: $\mathrm{CO}(r=0.05)$ and $\mathrm{NO}_{2}(r=0.09)$. Conclusions: Although the concentrations of air pollutants stay below the official limit, carbon monoxide and nitrogen dioxide affect the health of the child population of the Urban Area of Guadalajara. Although the monthly average mode and monthly mobile average does not exceed the established legislation, the registered maximums do exceed it; this indicates that although throughout most of the day there is no latent risk of popu-
\end{abstract}

(I) Instituto de Astronomía y Meteorología de la Universidad de Guadalajara. Guadalajara, Jalisco, México

(2) Departamento de Geografía y Ordenación Territorial del Centro Universitario de Ciencias Sociales y Humanidades de la Universidad de Guadalajara. Guadalajara, Jalisco, México

(3) Departamento de Salud Pública del Centro Universitario de Ciencias de la Salud de la Universidad de Guadalajara. Guadalajara, Jalisco, México 
yor parte del día no hay una exposición potencial de riesgo para la población a los contaminantes, sí existe exposición potencial de riesgo para la población al aire contaminado con concentraciones fuera de la norma durante algunas horas de ciertos días, lo cual puede influir de manera significativa en el incremento de infecciones agudas de las vías respiratorias superiores.

Palabras clave: infecciones agudas de las vías respiratorias; contaminantes atmosféricos;área urbana de Guadalajara,SIG; México lation exposure to the air pollutants, during some hours of certain days there is a risk for the population of breathing air contaminated with concentrations higher than the limit, which can cause the development of acute respiratory infections.

Key words: Acute respiratory infections, atmospheric polluting agents, urban Area of Guadalajara, GIS; Mexico
E 1 problema de los contaminantes emitidos en el área urbana de Guadalajara, como en la mayor parte de las grandes ciudades de México, se encuentra estrechamente relacionado con su esquema de desarrollo urbano, tecnológico e industrial.

Guadalajara, a partir de 1934, ha sido un importante centro comercial y manufacturero, lo que ha provocado un incremento de las vías de comunicación y el flujo migratorio; esto ha acentuado de manera directa e indirecta la creciente emisión de contaminantes atmosféricos desde entonces a la fecha.

En un inventario que realizó la SEMARNAP se advierte que las principales fuentes de contaminación atmosférica del área metropolitana de Guadalajara son el transporte, el suelo, la industria y los servicios. En ese mismo estudio se documentó que el sector transporte generó alrededor de $73.5 \%$ del total de las emisiones, en segundo lugar los suelos con $21.2 \%$, en tercer lugar los servicios con $4.2 \%$ y en cuarto lugar la industria con $1.1 \%{ }^{1}$

Diferentes estudios efectuados en otros países han mostrado nexos significativos entre los contaminantes atmosféricos monóxido de carbono (CO), dióxido de nitrógeno $\left(\mathrm{NO}_{2}\right)$, dióxido de azufre $\left(\mathrm{SO}_{2}\right)$, partículas menores de 10 micras $\left(\mathrm{PM}_{10}\right)$ y ozono $\left(\mathrm{O}_{3}\right)$ y las infecciones agudas de las vías respiratorias y otras enfermedades respiratorias en niños menores de 15 años; más aún, algunos contaminantes registrados por debajo de la norma oficial afectan también la salud de la población infantil. ${ }^{2-4}$

Esta investigación se basa en un análisis ecológico, que propuso Peter Haggett (1983), consistente en el estudio de las conexiones entre las variables ambientales y humanas dentro de espacios geográficos bien delimitados. ${ }^{5}$

El propósito es describir las correlaciones entre las variables ambientales (la contaminación atmosférica que emiten la naturaleza y los humanos) y los casos por infecciones agudas de las vías respiratorias de las unidades de medicina familiar y hospitales del Instituto Mexicano del Seguro Social (IMSS), las clínicas de medicina familiar del Instituto de Seguridad Social al Servicio de los Trabajadores del Estado (ISSSTE) y los centros de salud urbanos de la Secretaría de Salud Jalisco (SSJ) del área urbana de Guadalajara en el periodo 2000-2002, en relación con el espacio geográfico que delimita el área de influencia circunscrita a un radio de dos kilómetros de cada una de las estaciones de monitoreo atmosférico.

\section{Material y métodos}

El Departamento de Educación e Investigación Médica del Hospital de Pediatría CMNO, delegación estatal en Jalisco del Instituto Mexicano del Seguro Social (IMSS), evaluó el protocolo de esta investigación y se aprobó con el número de registro 2003-251-060 el día 27 de octubre del año 2003.

La información de las infecciones agudas de las vías respiratorias se obtuvo por medio de la Secretaría de Salud Jalisco y las clínicas, hospitales y centros de salud urbanos del IMSS, ISSSTE y SSJ del área urbana de Guadalajara. Se trabajó con la información mensual de los años 2000, 2001 y 2002 del número total de consultas por infecciones agudas de las vías respiratorias en niños menores de cinco años, de 28 unidades de medicina familiar y hospitales generales del IMSS, tres clínicas de medicina familiar del ISSSTE y 34 centros de salud urbanos de la SSJ, es decir, un total de 65 unidades de atención médica.

Las infecciones agudas de las vías respiratorias consideradas para el estudio correspondieron a las codificadas en la Décima Clasificación Internacional ${ }^{6}$ de las enfermedades como rinofaringitis aguda (J00), sinusitis aguda (J01), faringitis aguda (J02), amigdalitis aguda (J03), laringitis y traqueítis aguda (J04), laringitis 
obstructiva aguda y epiglotitis (J05), infecciones agudas de las vías respiratorias superiores de sitios múltiple o no especificados (J06). Asimismo, se incluyeron neumonía viral no clasificada en otra parte (J12), neumonía organismo no especificado (J18), bronquitis aguda (J20) y bronquiolitis aguda (J21), consideradas infecciones agudas de las vías respiratorias inferiores.

Por otro lado, el Instituto de Astronomía y Meteorología (IAM) de la Universidad de Guadalajara proporcionó la información de las modas, medias móviles y máximos mensuales de las concentraciones de los contaminantes atmosféricos de las ocho estaciones de monitoreo, ubicadas en el área urbana de Guadalajara, con base en los datos que suministró la Secretaría del Medio Ambiente para el Desarrollo Sustentable (SEMADES) del gobierno del estado de Jalisco, que realiza mediciones cada 10 minutos las 24 horas del día y los 365 días del año. En los casos en los que no fue posible obtener los registros de las concentraciones, se calculó la media anual.

Los parámetros medias móviles, modas y máximos se analizaron en tablas de distribución de frecuencias y se reconoció que los valores a los cuales estaba expuesta con mayor frecuencia la población estudiada en sus actividades diurnas (en las que desarrollan sus actividades los niños) eran los representados por las modas mensuales y medias móviles mensuales, mientras que los máximos sólo representaban menos de $0.2 \%$ de los datos. En consecuencia, se consideró que no eran representativos de la variable concentración de contaminantes, razón por la cual se decidió utilizar como índices de concentración la moda promedio mensual y las medias móviles mensuales de los contaminantes atmosféricos: monóxido de carbono, dióxido de azufre, dióxido de nitrógeno, partículas menores de 10 micras y ozono, de las ocho estaciones de vigilancia: Las Águilas, Atemajac, Centro, Loma Dorada, Miravalle, Oblatos, Tlaquepaque y Vallarta, de la Red Automática de Monitoreo Atmosférico (RAMA) del área urbana de Guadalajara, que opera la SEMADES del gobierno del estado de Jalisco.

Se localizaron las 65 unidades de atención médica y las ocho estaciones de monitoreo atmosférico por medio de las coordenadas Universal Transversa de Mercator (UTM), tomadas directamente en campo con un GPS (Sistema de Posicionamiento Global Satelital) portátil. Con base en la ubicación de las estaciones de vigilancia, se consideró que el área de estudio tenía un radio de influencia de dos kilómetros en cada una de ellas, unidos por una línea desde su parte exterior hasta cerrar el polígono, en el área urbana de Guadalajara.

La cartografía del área urbana de Guadalajara se obtuvo en formato digital del Departamento de Geo- grafía y Ordenación Territorial de la Universidad de Guadalajara, a partir del Instituto Nacional de Estadística, Geografía e Informática (INEGI), además de las cartas topográficas y ortofotos F13D65 Guadalajara Oeste, F13D66 Guadalajara Este, a escala 1:50 000 y actualizadas al año 1994.

\section{Procesamiento de la información}

Se creó el ambiente de trabajo en el programa IDRISI con las coordenadas extremas del área urbana de Guadalajara, así como la ubicación en coordenadas UTM de las estaciones de monitoreo atmosférico y las unidades de atención médica. Se digitalizó el límite del área de estudio y las principales vías de comunicación sobre las ortofotos georreferenciadas (figura 1).

Se aplicó la técnica de interpolación denominada "aproximación numérica" en la elaboración de los mapas (IDRISI) y para ello se diseñaron bases de datos con los valores de las modas mensuales y medias móviles mensuales para cada año y contaminante atmosférico, de tal forma que resultaron 216 mapas de modas mensuales y 216 de medias móviles mensuales interpoladas (figura 2). A partir de los mapas obtenidos se continuó con la extracción de las modas mensuales y medias móviles mensuales interpoladas para cada una de las 65 unidades de atención médica por medio de un listado procesado en IDRISI. Dichos resultados se trasladaron al programa Excel en bases de datos por mes y contaminante.

Se editaron los mapas de las modas mensuales y medias móviles mensuales interpoladas en el programa ARCVIEW con el fin de describir tendencias de la distribución espacial de los contaminantes atmosféricos (figura 2).

Asimismo, se diseñaron gráficas de las modas mensuales y medias móviles mensuales por contaminante y de las infecciones agudas de las vías respiratorias para el periodo de 2000 a 2002 en el programa Excel, con objeto de reconocer las tendencias.

Se elaboró la base de datos en Excel, integrada con 2324 registros y su respectivo numero total de consultas de infecciones agudas de las vías respiratorias de las unidades de atención médica (948 005 consultas) y 2324 datos de los resultados de la interpolación de cada uno de los contaminantes: monóxido de carbono, dióxido de nitrógeno, ozono, partículas menores de $10 \mathrm{mi}-$ cras y dióxido de azufre de las 65 unidades de atención médica (2000 a 2002).

Por otro lado, se calcularon en el programa SPSS coeficientes de correlación y determinación, regresión simple y múltiple, análisis de varianza (ANOVA) y prueba $t$ de las infecciones agudas de las vías respira- 


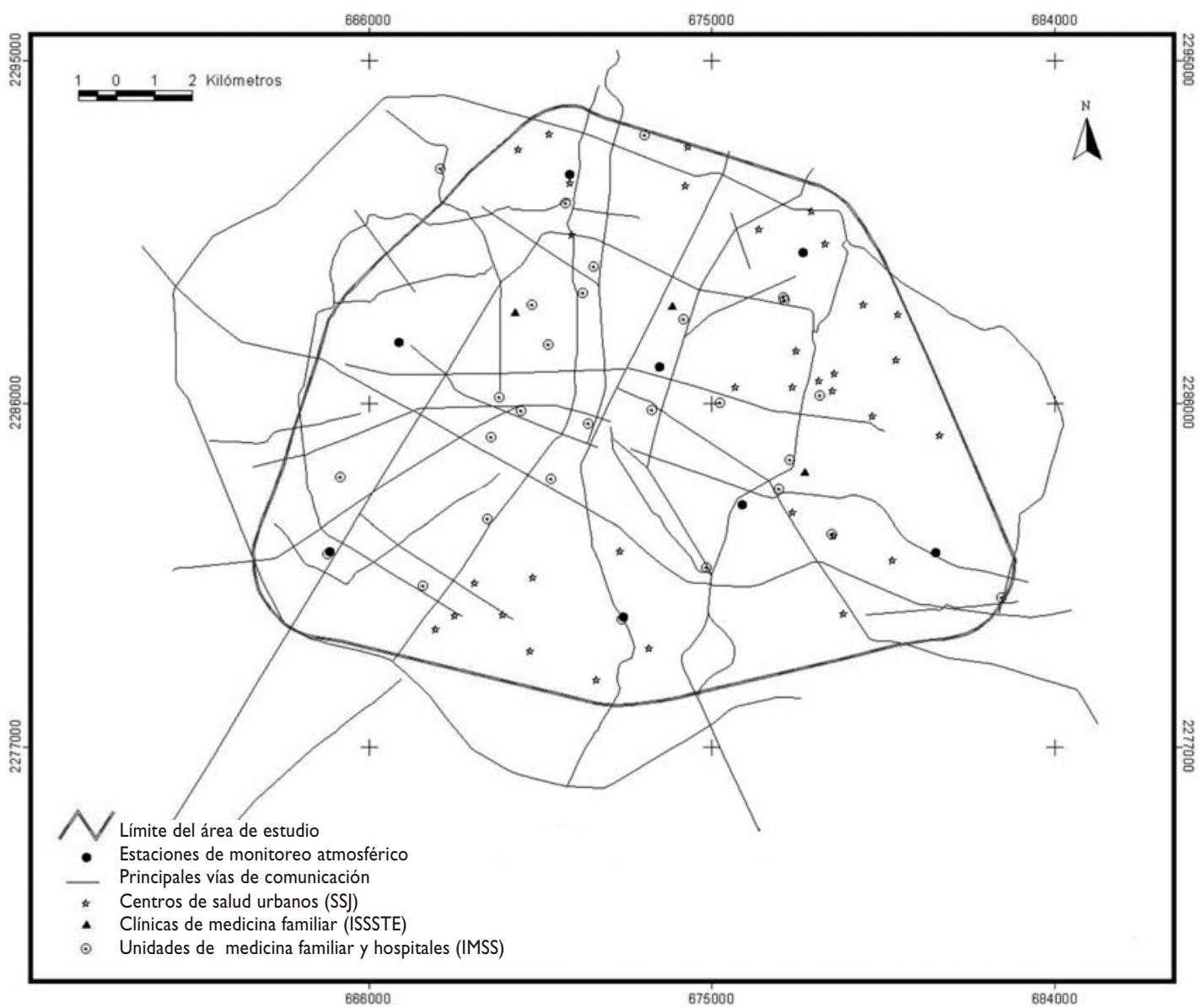

Figura I. Límites del ÁREA DE ESTUdio, PRINCIPALES Vías de COMUNICACIÓN, UBICACIÓN DE ESTACIONES DE MONITOREO ATMOSFÉRICO, CENTROS DE SALUd, CLíNICAS DE MEDICINA FAMILIAR DEL ISSSTE Y UNIDADES DE MEDICINA FAMILIAR Y HOSPITALES DEL IMSS (GuadalajARA, 2000-2002)

torias con el monóxido de carbono, dióxido de nitrógeno, ozono, partículas menores de 10 micras y dióxido de azufre, entre 2000 y 2002. El conjunto de pruebas estadísticas permitió determinar que las correlaciones con valores de $r \geq 0.05$ se consideraran estadísticamente significativas.

\section{Resultados}

\section{Comportamiento de los contaminantes atmosféricos}

El análisis de los máximos registrados muestra que 61 días de año 2000, 46 de 2001 y 51 de 2002 rebasaron la norma del CO (11 ppm). ${ }^{7}$ En el caso del $\mathrm{NO}_{2}(0.21$ ppm) ${ }^{7}$ los días que excedieron la norma fueron $18 \mathrm{de}$ 2000, 21 de 2001 y 27 de 2002. El ozono (0.11 ppm) ${ }^{7}$ presentó los siguientes días fuera de la norma: 75 días para el año 2000, 37 para 2001 y 78 para 2003. Las $P_{10}$ $\left(150 \mathrm{mg} / \mathrm{m}^{3}\right)^{7}$ son el contaminante que registra la máxima cantidad que supera la norma con 334 para 2000, 310 para 2001 y 311 para 2002. Por último, el SO $2(0.13$ ppm $)^{7}$ es el que menos rebasó el máximo permitido por la norma con cuatro días para el año 2000 y 2001 y 10 días para 2002 (cuadro I).

La distribución de los niveles de concentraciones de las modas mensuales y medias móviles mensuales interpoladas de los contaminantes atmosféricos en el área urbana de Guadalajara difiere en grado considerable de un contaminante a otro, debido a que al- 

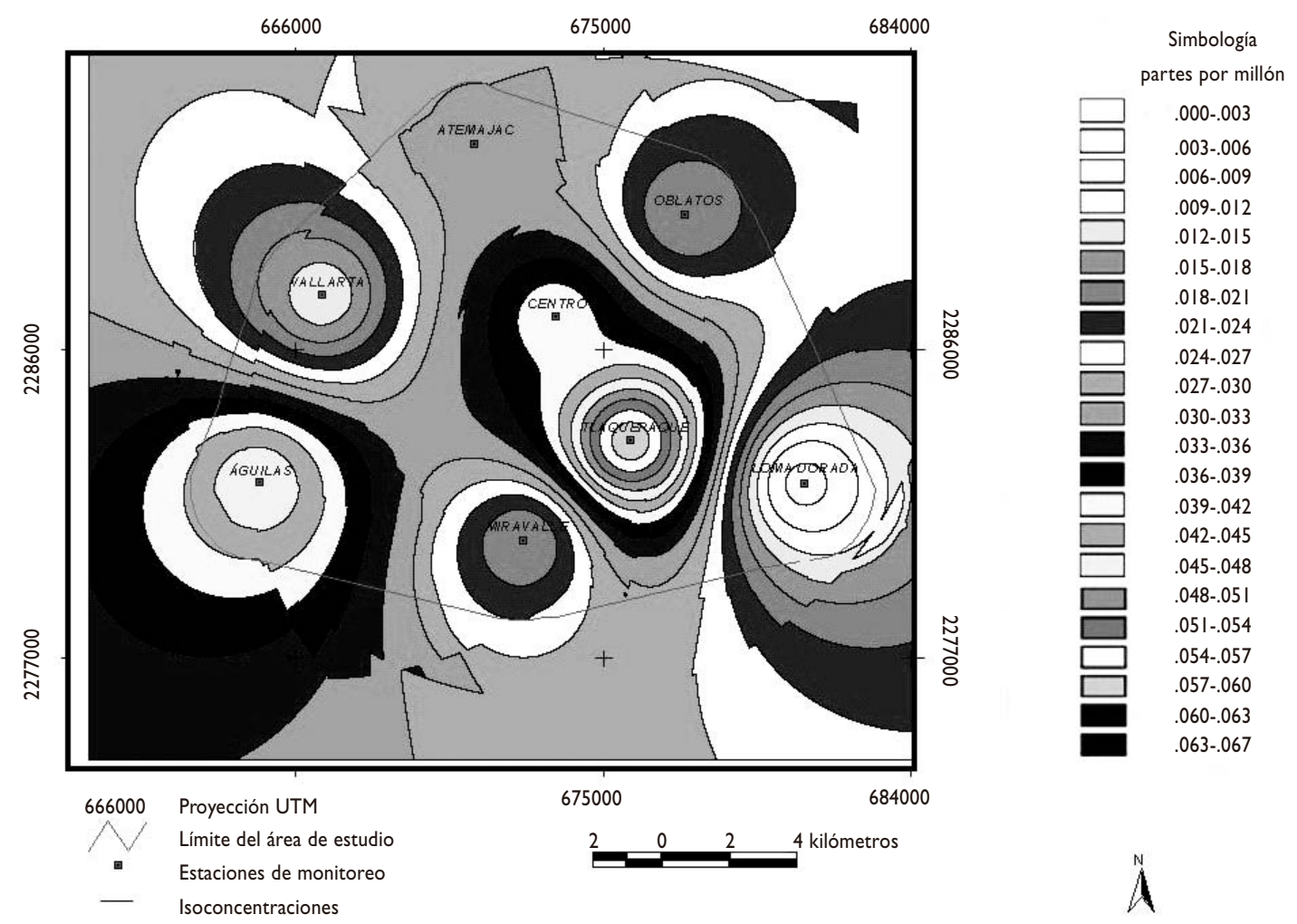

Figura 2. Tendencias de la distribución espacial del dióxido de nitrógeno (Guadalajara, mayo 2002)

\section{Cuadro I}

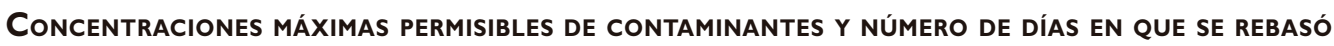
LA norma en el Área urbana de Guadalajara (2000-2002)

\begin{tabular}{|c|c|c|c|c|c|}
\hline \multicolumn{6}{|c|}{ Valores límite } \\
\hline Monóxido de carbono $(\mathrm{CO})^{\S}$ & II ppm (8 hrs) & I vez al año & $61 *$ & $46 *$ & 51 \\
\hline Dióxido de nitrógeno $\left(\mathrm{NO}_{2}\right)^{\#}$ & 0.21 ppm (I hr) & I vez al año & 18 & 21 & 27 \\
\hline Ozono $\left(\mathrm{O}_{3}\right)^{\&}$ & 0.11 ppm (I hr) & I vez cada 3 años & 75 & 37 & 78 \\
\hline Partículas, fracción respirable $\left(\mathrm{PM}_{10}\right)^{\neq}$ & $150 \mathrm{mg} / \mathrm{m}^{3}$ (24 hrs) & I vez al año & $334^{\ddagger}$ & $310^{\ddagger}$ & $311 \ddagger$ \\
\hline Dióxido de azufre $\left(\mathrm{SO}_{2}\right)^{\infty}$ & 0.13 ppm (24 hrs) & I vez al año & $4^{\ddagger}$ & $4^{\ddagger}$ & $10^{\ddagger}$ \\
\hline 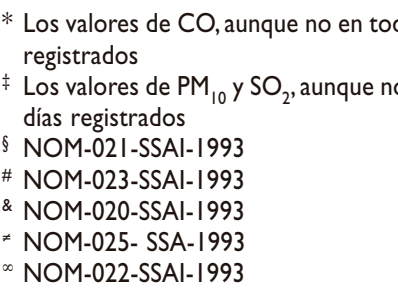 & $\begin{array}{l}\text { asos se presentaron } \\
\text { os los casos se prese }\end{array}$ & $\begin{array}{l}24 \text { horas continuas, sí re } \\
\text { ranuas, }\end{array}$ & $\begin{array}{l}\text { la nor } \\
\text { aron la }\end{array}$ & $\begin{array}{l}\text { cierto } \\
\text { den c }\end{array}$ & $\begin{array}{l}\text { s de los días } \\
\text { entos de los }\end{array}$ \\
\hline
\end{tabular}


gunas de las estaciones de monitoreo registran con regularidad niveles más bajos que otras, así como diferentes tipos de contaminantes (cuadro II).

En cuanto a la distribución de las infecciones agudas de las vías respiratorias en el área urbana de Guadalajara, se analizaron 948005 consultas en total; sin embargo, existe una marcada diferencia en relación con el número de consultas, esto es, mientras en una clínica se registraron tres consultas mensuales, en otra se notificaron 3 942, lo cual presenta una alta dispersión respecto del promedio (cuadro III).

\section{Cuadro II}

Concentración PROMEdio de las MOdAs DE CONTAMINANTES Y PROMEDIO DEL NÚMERO DE INFECCIONES AGUDAS DE LAS VÍAS RESPIRATORIAS en el Área urbana de Guadalajara (2000-2002)

\begin{tabular}{lcccr} 
Contaminante & Promedio & $\begin{array}{c}\text { Desviación } \\
\text { estándar }\end{array}$ & $\begin{array}{c}\text { Valor } \\
\text { mínimo }\end{array}$ & $\begin{array}{c}\text { Valor } \\
\text { máximo }\end{array}$ \\
$\mathrm{CO}$ & 1.170 & 0.340 & 0.246 & $3.81 \mathrm{I}$ \\
\hline $\mathrm{NO}_{2}$ & 0.025 & 0.008 & 0.000 & 0.066 \\
\hline $\mathrm{O}_{3}$ & 0.010 & 0.006 & 0.000 & 0.062 \\
\hline $\mathrm{PM}_{10}$ & 37.892 & 31.117 & 2.310 & 499.730 \\
\hline $\mathrm{SO}_{2}$ & 0.007 & 0.002 & 0.001 & 0.020 \\
\hline Infecciones respiratorias & 408 & 556 & 3 & 3942
\end{tabular}

El CO, $\mathrm{NO}_{2}, \mathrm{O}_{3}$ y $\mathrm{SO}_{2}$ se miden en partes por millón (ppm); las $\mathrm{PM}_{10}$ en microgramo por metro cúbico $\left(\mu \mathrm{g} / \mathrm{m}^{3}\right)$

Cuadro III

Matriz de correlaciones de contaminantes ATMOSFÉRICOS E INFECCIONES AGUDAS DE LAS VÍAS RESPIRATORIAS EN NIÑOS MENORES DE CINCO AÑOS (Guadalajara, 2000-2002)

\begin{tabular}{llllll} 
& Infecciones respiratorias & $\mathrm{CO}$ & $\mathrm{NO}_{2}$ & $\mathrm{O}_{3}$ & $P M_{10}$ \\
$\mathrm{CO}$ & 0.05 & & & & \\
\hline$P$ & 0.01 & & & & \\
\hline $\mathrm{NO}_{2}$ & 0.09 & 0.12 & & & \\
\hline$P$ & 0.00 & 0.00 & & & \\
\hline $\mathrm{O}_{3}$ & 0.01 & -0.17 & 0.05 & & \\
\hline$P$ & 0.80 & 0.00 & 0.02 & & \\
\hline $\mathrm{PM}_{10}$ & 0.02 & 0.21 & 0.15 & -0.03 & \\
\hline$P$ & 0.33 & 0.00 & 0.00 & 0.17 & \\
\hline $\mathrm{SO}_{2}$ & 0.04 & 0.15 & 0.19 & 0.08 & 0.15 \\
\hline$P$ & 0.09 & 0.00 & 0.00 & 0.00 & 0.00 \\
$P=$ significancia & & & & & \\
& & & & &
\end{tabular}

\section{Comportamiento de los contaminantes atmosféricos en relación con las infecciones agudas de las vías respiratorias}

Con este estudio se definió la correlación entre los contaminantes atmosféricos $\left(\mathrm{CO}, \mathrm{NO}_{2}, \mathrm{O}_{3^{\prime}} \mathrm{PM}_{10} \mathrm{y} \mathrm{SO}_{2}\right)$ con el número total de consultas por infecciones agudas de las vías respiratorias en niños menores de cinco años, de centros de salud, clínicas y hospitales del IMSS, ISSSTE y SSJ del área urbana de Guadalajara durante 2000, 2001 y 2002.

En esta aproximación a los efectos de los contaminantes atmosféricos que inciden en la salud de la población del área urbana de Guadalajara, la correlación de los contaminantes mostró vínculos directos estadísticamente significativos $(r \geq 0.05)$ con las infecciones agudas de las vías respiratorias en los niños menores de cinco años de $r=0.05$ para el CO y $r=0.09$ para el $\mathrm{NO}_{2}$; por su parte, en los contaminantes $\mathrm{O}_{3}(r=0.01)$, $\mathrm{PM}_{10}(r=0.02)$ y SO${ }_{2}(r=0.04)$ no se advierte relación alguna con las infecciones agudas de las vías respiratorias (cuadro III).

El análisis de los resultados evidenció que la presencia de un contaminante en la atmósfera se correlaciona con la de otros; en el caso del CO se observa una correlación significativa en relación con el $\mathrm{NO}_{2}$ $(r=0.12), \mathrm{O}_{3}(r=-0.17), \mathrm{PM}_{10}(r=0.21)$ y SO $\mathrm{SO}_{2}(r=0.15)$. De igual forma, el $\mathrm{NO}_{2}$ manifiesta una correlación significativa con los contaminantes $\mathrm{O}_{3}(r=0.05), \mathrm{PM}_{10}(r=$ $0.15)$ y $\mathrm{SO}_{2}(r=0.19)$. Asimismo, el $\mathrm{SO}_{2}$ encuentra correlación relevante con $\mathrm{O}_{3}(r=0.08)$ y $\mathrm{PM}_{10}(r=0.15)$. Esta sinergia explicable por la combinación de dos o más especies contaminantes podría potenciar el desarrollo de infecciones agudas de las vías respiratorias (cuadro III).

\section{Discusión}

La exposición a los contaminantes atmosféricos representa un riesgo para la población que vive en las grandes ciudades de América Latina, ${ }^{8}$ y el área urbana de Guadalajara no está exenta de ello; los niños y los ancianos son la población más vulnerable y presentan mayores riesgos en su salud debido a una exposición más intensa a los contaminantes atmosféricos.

Las ocho estaciones de la Red Automática de Monitoreo Atmosférico (RAMA) de la zona metropolitana de Guadalajara (Las Águilas, Atemajac, Centro, Loma Dorada, Miravalle, Oblatos, Tlaquepaque y Vallarta), operan y generan información cada 10 minutos, las 24 horas del día y los 365 días del año. Este sistema permite obtener información confiable, de forma oportuna y en un formato accesible para proce- 
sarse en programas de cómputo. En el caso particular de este estudio, antes de utilizar las modas promedio mensuales como variable de análisis, se cotejó toda la información que suministraron las estaciones de monitoreo cada 10 minutos y se observó que la población se mantenía expuesta con una mayor frecuencia al valor establecido por la moda, mayor que el de la media aritmética. Al analizar los valores mensuales de la moda y la media aritmética resultó evidente que los valores extremos afectaban en gran proporción la media aritmética y dichos valores no se sostenían, sino que eran muy esporádicos y no reflejaban los valores a los que estaba expuesta la población la mayor parte del día; por lo tanto, se recurrió en este caso a la moda. Sin embargo, evaluar las correlaciones con las medias móviles mensuales arrojó resultados similares.

Los resultados de esta investigación muestran que la frecuencia de infecciones agudas de las vías respiratorias se correlaciona de manera directa con la presencia de $\mathrm{CO}$ y $\mathrm{NO}_{2}$; tal nexo, aunque débil, es significativo.

Aunque los resultados muestran que las modas promedio mensuales no rebasan la normatividad establecida, los máximos registrados sí lo hacen en los días mostrados en el cuadro I; esto permite indicar que si bien en la mayoría del día no hay una exposición potencial de riesgo para la población a los contaminantes analizados, sí existe durante algunos momentos de ciertos días riesgo de la población a respirar el aire contaminado con concentraciones que superan la norma, lo cual puede alterar el desarrollo de infecciones agudas de las vías respiratorias.

En el caso del análisis de las modas promedio mensuales, se observó que los contaminantes atmosféricos que incidieron en la salud de los niños menores de cinco años del área urbana de Guadalajara fueron $\mathrm{CO}_{~ \mathrm{NO}_{2}}$ los cuales presentaron una correlación significativa con los casos de infecciones agudas de las vías respiratorias.

Cabe mencionar que en algunas ocasiones las estaciones de monitoreo se descomponen y no es posible registrar los niveles de concentración de los contaminantes; cuando esto sucede es preciso decidir y considerar otras alternativas para disponer de esta información; en este caso se tomó la moda promedio anual para sustituir el valor faltante de la moda mensual o la media móvil mensual en algunos meses del periodo trabajado, o ambos parámetros.

Por otro lado, la distribución de las estaciones de monitoreo atmosférico en la zona metropolitana de Guadalajara no permite obtener información de un lugar específico, aun si se toman en consideración los dos kilómetros de radio que emplea la SEMADES como área de influencia de cada estación de monitoreo; pese a ello, existen grandes zonas que exceden esta área de influencia (figura 1); por esa razón, se elaboraron mapas de interpolación de las modas mensuales y medias móviles mensuales de cada uno de los contaminantes atmosféricos, lo que permitió obtener información de concentraciones de cada una de las unidades de atención médica.

Es importante considerar que las instituciones (IMSS, ISSSTE y SSJ) que ofrecen los servicios de salud tienen su propia estructura operativa y atienden a la población de los cuatro municipios (Guadalajara, Tlaquepaque, Tonalá y Zapopan) que integran la zona metropolitana de Guadalajara; en muchos casos, los usuarios que demandan estos servicios viven muy lejos de las unidades en que se atienden y están expuestos las 24 horas del día a los niveles elevados de concentración de los contaminantes, lo que puede trastocar los resultados.

La mayoría de los estudios sobre correlaciones entre la exposición a contaminantes atmosféricos y el número de consultas por enfermedades respiratorias se ha llevado a cabo en lugares que presentan concentraciones de contaminación elevadas; empero, estudios realizados en lugares con bajos niveles de contaminantes también han identificado una vinculación positiva. ${ }^{9}$

Hernández-Cadena y colaboradores, ${ }^{9}$ en un estudio de consultas a urgencias por enfermedades respiratorias en dos hospitales del IMSS, respecto de los niveles de contaminación del aire en Ciudad Juárez, registraron un total de 12721 consultas de niños menores de 15 años por infecciones agudas de las vías respiratorias.El mayor número de consultas por enfermedades respiratorias en niños se presentó en el grupo de uno a cuatro años, con cerca de $50 \%$ de los casos, seguido por el grupo de niños menores de un año. Las correlaciones de los contaminantes entre los dispositivos de vigilancia utilizados para cuantificar la exposición media diaria fluctuaron entre 0.85 y $0.99(p<0.01)$ para $\mathrm{O}_{3}$ y entre 0.32 y $0.91(p<0.05)$ para $\mathrm{PM}_{10}$. En este estudio se hallaron correlaciones mucho menos elevadas y significativas para el $\mathrm{O}_{3}$ y $\mathrm{PM}_{10^{\prime}}$ por lo que se manifiesta una contradicción en el comportamiento de estos dos contaminantes respecto de los autores mencionados.

Estudios epidemiológicos demuestran que la exposición a diferentes contaminantes, incluso a niveles situados por debajo de la norma, se vinculan con el deterioro de la función pulmonar, así como con otras enfermedades respiratorias en niños y adolescentes. En una investigación realizada en La Habana que relacionó la contaminación atmosférica, asma bronquial e in- 
fecciones respiratorias agudas en menores de edad, se evidenció que la exposición al $\mathrm{SO}_{2}$ se vinculó con un incremento del número de visitas de urgencia por infecciones agudas de las vías respiratorias. En dicho estudio, el incremento de los niveles de contaminantes atmosféricos, en particular de humo, se relacionó con un aumento de las visitas de urgencia hospitalaria por infecciones agudas de las vías respiratorias en niños menores de 14 años de edad. Los niveles de otros contaminantes del aire $\left(\mathrm{PM}_{10}\right.$ y SO 2$)$ también se vincularon con el número de visitas a urgencias. Sus resultados concluyen que el humo, el SO 2 y las $\mathrm{PM}_{10}$ poseen un nexo con el número de consultas por infecciones agudas de las vías respiratorias; ello coincide con otros estudios, los cuales refieren un efecto adverso de los contaminantes atmosféricos sobre el sistema respiratorio del individuo. Al final, la exposición a la mezcla de los contaminantes del aire incrementa el riesgo de infecciones respiratorias..$^{10}$ Estas dos conclusiones concuerdan con los resultados evidenciados en el presente estudio en el que existe correlación de ciertos contaminantes con el número de consultas por infecciones agudas de las vías respiratorias y los efectos sinérgicos de la presencia simultánea de varios contaminantes, lo que magnifica su influencia en las infecciones agudas de las vías respiratorias.

Pocos estudios han evaluado el efecto de los contaminantes sobre las infecciones respiratorias agudas; los resultados de este trabajo sugieren que los niveles permitidos en las normas internacionales no protegen a los niños del efecto adverso que producen los contaminantes del aire sobre las funciones respiratorias. Los resultados del estudio de La Habana, así como los de este protocolo, sugieren que aun los bajos niveles de contaminación del aire en las ciudades son dañinos para el tracto respiratorio de los menores de edad, y que ello se relaciona con el aumento del riesgo de padecer infecciones agudas de las vías respiratorias.

Un estudio de Téllez-Rojo y colaboradores, ${ }_{11}^{11}$ en el IMSS del suroeste del Distrito Federal, determinó la relación entre el número de consultas por infecciones respiratorias con los niveles diarios de los principales contaminantes que aquejan a la ciudad. Se utilizaron las mediciones diarias de $\mathrm{O}_{3}, \mathrm{SO}_{2}$ y $\mathrm{NO}_{2}$ de la estación Pedregal de la Red Automática de Monitoreo Ambiental del Distrito Federal. Durante el periodo del estudio se presentó un total de 19139 consultas de niños menores de 15 años por infecciones agudas de las vías respiratorias. En $74.3 \%$ de los días se rebasó la norma mexicana para el $\mathrm{O}_{3^{\prime}}$ mientras que la norma para el $\mathrm{SO}_{2}$ quedó muy lejos de alcanzarse; por último, la norma de la Organización Mundial de la Salud para el promedio de 24 horas del $\mathrm{NO}_{2}$ es de 0.08 ppm, que sólo se rebasó tres días del año. En esta investigación se encontraron valores diferentes respecto de los que informaron los autores referidos, ya que para el caso del $\mathrm{O}_{3}$ sólo en $17.35 \%$ de los días se superó la norma durante el periodo de estudio, para el $\mathrm{SO}_{2}$ se presentaron 14 días fuera de la norma durante los tres años analizados y para el $\mathrm{NO}_{2}$, con norma de $0.21 \mathrm{ppm}$, se rebasó el parámetro en $6 \%$ de los días.

Estos mismos autores sugieren un posible nexo entre los días con niveles elevados del contaminante $\mathrm{O}_{3}$ y los incrementos del número de visitas por infecciones agudas de las vías respiratorias. Asimismo, demuestran que los padecimientos de vías superiores presentan una tendencia creciente con incremento de $\mathrm{O}_{3}$, a medida que aumenta el tiempo entre la exposición y la aparición de la enfermedad; en lo que se refiere al $\mathrm{NO}_{2}$, las relaciones también resultaron sumamente significativas; no obstante, se perdió la tendencia creciente detectada para el $\mathrm{O}_{3}$. Sus resultados sugieren que la exposición de los menores de 15 años al $\mathrm{O}_{3} \mathrm{y}$ $\mathrm{NO}_{2}$ incide en grado notorio sobre el número de consultas ocasionadas por motivos respiratorios en esta zona de la Ciudad de México. En el caso particular del área urbana de Guadalajara se obtuvo una influencia del $\mathrm{NO}_{2}$ sobre el número de consultas por infecciones agudas de las vías respiratorias, lo que coincide con lo presentado en el suroeste de la Ciudad de México, pero difiere en el comportamiento para el $\mathrm{O}_{3^{\prime}}$ que no es significativo en el presente estudio.

En 1948, los niveles extremadamente altos de contaminación del aire se vincularon con episodios de mortalidad excesiva en Donora, Pennsylvania, Estados Unidos (EU), al igual que en Londres, Inglaterra, en 1952, y en Mouse Valley, Bélgica, en 1930. Estos episodios se caracterizaron por elevados niveles de partículas, dióxido de azufre y mezclas. En un estudio realizado en la Ciudad de México se observó un incremento de la mortalidad vinculado de manera independiente con ozono, dióxido de azufre y partículas totales en suspensión. Cuando se consideraron los tres contaminantes de modo simultáneo en el mismo modelo, sólo las partículas totales en suspensión se relacionaron con la mortalidad. ${ }^{12}$ Aunque en este análisis no se presentó ni evaluó la mortalidad, se confirmó que la presencia simultánea de varios contaminantes eleva la presencia de las afecciones respiratorias.

Es relevante mencionar la existencia de informes de casos de muerte por intoxicación por monóxido de carbono dentro del vehículo y en los hogares. ${ }^{2}$ Asimismo, durante la Guerra del Golfo se quemaron pozos y refinerías de aceite; en consecuencia, Kuwait se expuso 
a este gas tóxico y se reconoció un considerable aumento de demandantes de atención médica por irritación en las vías respiratorias superiores. ${ }^{3}$

Otros estudios hacen referencia a una relación del incremento de hospitalizaciones por enfermedad respiratoria atribuidas al monóxido de carbono. ${ }^{13}$ De igual forma, al ser respirado por los fumadores se advirtió una tendencia al incremento de la concentración del monóxido de carbono conforme aumentaba el número diario de cigarrillos consumidos por día y el tiempo del hábito. ${ }^{14}$ Esto confirma la correlación significativa obtenida con esta investigación entre las infecciones agudas de las vías respiratorias en los niños menores de cinco años y el monóxido de carbono.

El dióxido de nitrógeno es otro contaminante que debe estudiarse, ya que se ha relacionado en grado relevante con el número de consultas por infecciones respiratorias. ${ }^{11,15}$ Ello es muestra del vínculo obtenido en esta investigación de las infecciones agudas en las vías respiratorias superiores con el dióxido de nitrógeno.

Asimismo, se intentó definir la relación entre los contaminantes ozono, dióxido de azufre y partículas menores de 10 micras con infecciones agudas de las vías respiratorias y no se identificó correlación alguna, a diferencia de otros estudios en los que se definió una correlación de importancia. ${ }^{11,16-18}$

Cabe mencionar que este trabajo de investigación se realizó con los Sistemas de Información Geográfica (SIG), mediante las nuevas tecnologías para el tratamiento de la información, y se empleó un enfoque geográfico, desde el punto de vista de la distribución espacial de las concentraciones de los contaminantes atmosféricos y las infecciones agudas de las vías respiratorias; de esa forma se obtuvieron correlaciones significativas de las infecciones agudas de estas vías con dos de los cinco contaminantes estudiados $(\mathrm{CO}$, $\mathrm{NO}_{2}$ ) durante un periodo de tres años.

En este sentido, con base en los resultados de la presente investigación, es posible afirmar que los contaminantes monóxido de carbono y dióxido de nitrógeno afectan la salud de la población. De igual modo, a partir de los mapas de isoconcentraciones se puede inferir la distribución de los niveles más altos de concentraciones de estos contaminantes $\mathrm{y}$, por consiguiente, definir las áreas expuestas y por supuesto la población en riesgo del área urbana de Guadalajara (figura 2).

Lo anterior indica que falta todavía mucho por hacer, por ejemplo, realizar inventarios periódicos de las principales fuentes emisoras de estos contaminantes atmosféricos, sobre todo en aquellas áreas definidas con altas concentraciones, e investigar a la población expuesta a los más altos índices de concen- tración de los contaminantes. Todo ello tiene la finalidad de aplicar programas y concretar acciones que conduzcan a disminuir los niveles de concentraciones de los contaminantes atmosféricos. Es posible formular las siguientes conclusiones:

1. A pesar de que los niveles de concentración se mantienen por debajo de la norma oficial, en la cual se establecen criterios para evaluar la calidad del aire de los contaminantes, el monóxido de carbono y el dióxido de nitrógeno alteran la salud de la población infantil del área urbana de Guadalajara.

2. Aunque los resultados muestran que las modas mensuales y medias móviles mensuales no rebasan la normatividad establecida, los máximos registrados sí lo hacen. En consecuencia, puede indicarse que si bien en la mayor parte del día no hay un riesgo latente de exposición de la población a los contaminantes analizados, sí existe durante algunas horas de ciertos días riesgo de la población a respirar el aire contaminado con concentraciones que exceden la norma, lo cual puede propiciar el desarrollo de infecciones agudas de las vías respiratorias superiores.

3. Se sugiere continuar con este tipo de investigación para evaluar de manera más precisa la influencia de contaminantes en el desarrollo de infecciones agudas de las vías respiratorias, a través de la conformación de un grupo multidisciplinario de especialistas de la salud y calidad del aire.

\section{Agradecimientos}

Se agradece la colaboración del personal de la Secretaría del Medio Ambiente para el Desarrollo Sustentable (SEMADES) del gobierno del estado de Jalisco por el acceso a los datos de la Red Automática de Monitoreo Atmosférico. Asimismo, se agradece al personal del Instituto de Astronomía y Meteorología de la Universidad de Guadalajara por el trabajo estadístico de la información de las concentraciones de los contaminantes atmosféricos de las ocho estaciones de monitoreo de la RAMA, y al departamento de Geografía de la Universidad de Guadalajara por su colaboración en el empleo de las ortofotos del área urbana de Guadalajara en formato digital.

\section{Referencias}

I. Gobierno del Estado de Jalisco, Secretaría del Medio Ambiente Recursos Naturales y Pesca, Secretaría de Salud. Programa para el mejoramiento de la calidad del aire en la Zona Metropolitana de Guadalajara 1997-200 I. México: Gobierno del Estado de Jalisco, 1997. 
2. Ruszkiewicz A, Boer B, Robertson S. Usual presentation of death due to carbon monoxide poisoning. A report of two cases. Am J Forensic Med Pathol 1997; 18:18I-184.

3. Osman Y. Environmental surveys conducted in the Gulf region following the Gulf War to identify possible neurobehaviral consequences. Environmental Research 1997;73(I-2):207-2I0.

4. Álvarez-Sintes R, Álvarez-Castro MR, Toledo-Vila H.Asociación entre manifestaciones respiratorias atípicas y contaminantes primarios de la atmósfera. Revista Cubana Med Gen Integr 1997;13(3):212-22I.

5. Jensen AH. Geografía, historia y conceptos. Barcelona:Vicens Vives, 1987. 6. Organización Mundial de la Salud. Clasificación Estadística Internacional de Enfermedades y Problemas Relacionados con la Salud, Décima Revisión, publicación científica no. 554. Washington: DC/OPS, 1995.

7. Diario Oficial de la Federación. NOM-020-SSAI-1993, 30 de octubre del 2002, NOM-02I-SSAI-1993, 23 de diciembre de 1994, NOM-022SSAI-1993, 23 de diciembre 1994, NOM-023-SSAI-1993, 23 de diciembre de 1994, NOM-025-SSAI-1993, 23 de diciembre 1994. 8. Belmar R, HofmeisterVA, Michaels D, Moreno A, Romieu I. Epidemiología ambiental. Un proyecto para América Latina y el Caribe. Organización Panamericana de la Salud 1994:37-46.

9. Hernández-Cadena L, Téllez-Rojo MM, Sanín-Aguirre LH, LascañaNavarro M, Campos A, Romieu I. Relación entre consultas a urgencias por enfermedad respiratoria y contaminación atmosférica en Ciudad Juárez, Chihuahua. Salud Publica Mex 2000;42:288-297.

10. Romero-Placeres M, Más-Bermejo P, Lacasaña-Navarro M,TéllezRojo MM, Aguilar-Valdés J, Romieu I. Contaminación atmosférica, asma bronquial e infecciones respiratorias agudas en menores de edad de La Habana. Salud Publica Mex 2004;46:222-233.

I I. Tellez-Rojo MM, Romieu I, Peña MP, Ruiz-Velasco S, Meneses-

González F, Hernández-Avila M. Efectos de la contaminación ambiental sobre las consultas por infecciones respiratorias en niños de la Ciudad de México. Salud Publica Mex 1997;39:5।3-522.

12. Lacasaña-Navarro M, Aguilar-Garduño C, Romieu I. Evolución de la contaminación del aire e impacto de los programas de control en tres megaciudades de América Latina. Salud Publica Mex 1999;41:203-215. 13. Ocaña-Servín H, Escobedo V, García-Rosas R. Contaminación atmosférica e hiperreactividad bronquial en la edad pediátrica. Efecto protector del ketotifeno. Revista Mexicana de Pediatría 1991;58:93-100. 14. Quezada R, Martínez-Rossier LA, Hernández-Garduño E, CatalánVázquez M, Quezada E et al. Comparación de los niveles de monóxido de carbono espirado por los transeúntes de un área urbana y de un área rural. Revista del Instituto Nacional de Enfermedades Respiratorias 1997;10:13-17.

15. Hernández-Garduño E, Pérez-Neria J, Paccagnella AM, Piña-García MA, Munguía-Castro M, Catalán-Vázquez M, et al.Air pollution and respiratory health in México City. Occup Environ Med 1997:39:299-307. 16. Romero M, Bermejo P, Lacasaña M,Téllez M,Aguilar-Valdés J, et al. Contaminación atmosférica, asma bronquial e infecciones respiratorias agudas en menores de edad de La Habana. Salud Publica Mex 2004:46:222-233.

17. Molina E, Brown L, Prieto V, Bonet M, Cuéllar L. Crisis de asma y enfermedades respiratorias agudas, contaminantes atmosféricos y variables meteorológicas en Centro Habana. Rev Cubana Med Gen Integr 2001;17:10-20.

18. Cuesta O,Wallo A, Collazo A, Arriba A, González M, Labrador R. Enfermedades respiratorias $y$ calidad del aire en la zona de la Ribera Este de la Bahía de La Habana. Centro de Contaminación y Química Atmosférica Ciudad de La Habana. Conferencia Internacional Trópico 2004. Palacio de Convenciones La Habana Cuba.Abril del 2004. 